\title{
Alteration in 11 Beta-Hydroxysteroid Dehydrogenase Type-2 (HSD11B2) Gene as A Potential Candidate Parameter for Early Detection of Intrauterine Growth Restriction (IUGR) Events
}

\author{
Louis Fabio Jonathan Jusni, Patricia, Brigitte Leonie Rosadi \\ Faculty of Medicine and Health Science, Atma Jaya Catholic University of Indonesia, Jakarta, Indonesia
}

Intrauterine Growth Restriction (IUGR) incidence in Indonesia ranks in the top 10 of the highest in Asia. It is the main perinatal death cause. IUGR also impairs fetal neurodevelopment, which can affect the development of children until later ages. Lack of $11 \beta$-hydroxysteroid dehydrogenase type-2 (11ß-HSD2) enzyme is influenced by changes in the coding gene, HSD11B2, one of IUGR's causes. The main diagnostic method of IUGR at this time is by using Doppler ultrasound. However, Doppler ultrasound has several limitations as many cases are not detected. Its clinical predictive value in various women is poor, as Doppler ultrasound is not recommended for use in the first trimester, detection of abnormalities in the second trimester seems to be too late for helpful interventions. The study aim is to present an overview concerning HSD11B2 gene alteration in an non-invasive prenatal testing (NIPT) as a possible diagnostic parameter for early detection in IUGR infants. This literature review is based on selected articles and studies taken from the Pubmed, Proquest, and EBSCO databases. A total of 4 studies reported the tendency for DNA methylation and decreased expression of the HSD11B2 gene in IUGR cases. Changes in the HSD11B2 gene have the potential to become a diagnostic parameter in the early detection of infants with IUGR. Further study and investigation of this possibility are needed.

Keywords: intrauterine growth restriction, HSD11B2, early detection, diagnostic, non-invasive prenatal testing

\section{Introduction}

Intrauterine growth restriction (IUGR), or fetal growth retardation according to The American College of Obstetricians and Gynecologists (ACOG), is a fetal condition with an estimated body weight less than $10^{\text {th }}$ percentile during pregnancy. ${ }^{1}$ In developing countries, the incidence of IUGR is known to be 6 times higher than in developed countries. Seventy-five percent of IUGR cases are in the Asian continent. Indonesia remains as the top 10 countries with the most IUGR incidents in Asia. ${ }^{2}$

IUGR is the leading cause of perinatal death. It also can cause stillbirth or premature fetal death and premature birth. The incidence of stillbirth is higher in undetected IUGR. Infants born with IUGR have a risk of having shortterm complications, including Low Appearance, Pulse,

Date of submission: June 28, 2021

Last Revised: September 12, 2021

Accepted for publication: September 14, 2021

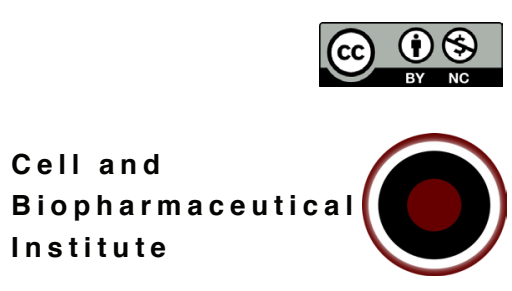

Corresponding Author:

Louis Fabio Jonathan Jusni

Faculty of Medicine and Health Science

Atma Jaya Catholic University of Indonesia

Jl. Pluit Selatan Raya No.19, Jakarta, Indonesia

e-mail: louisjusni@gmail.com 
Grimace, Activity, and Respiration Score (APGAR Score), hypoxia, hypothermia, and hypoglycemia. ${ }^{3,4}$ While longterm complications that can occur are inhibition of physical growth and brain development. Infants' neurodevelopmental impairment because of IUGR can result in a lack of language, memory, visuomotor, and executive function skills, affecting their academic performance in the future. ${ }^{4}$

Currently, the diagnosis of IUGR is achieved by Doppler ultrasound as the main diagnostic tool and examination of Adverse Pregnancy Outcome (APO). Two antenatal devices diagnostic accuracy has proved to be inadequate in some cases. ${ }^{5}$ Doppler ultrasound's clinical predictive value in various women is poor. Moreover, discovering abnormalities in the second trimester, which is the recommended time for Doppler ultrasound testing, seems to be too late for helpful interventions. ${ }^{6}$ The detection of IUGR cases had not improved since the late 1990s, and about half of the pregnancies with IUGR were undetected. ${ }^{7}$ Also, ultrasonography is also very operator-dependent, so that the interpretation of the results may differ. ${ }^{8}$

To examine IUGR, a safe alternative method, called non-invasive prenatal testing (NIPT) or somewhile called non-invasive prenatal screening (NIPS), can be conducted. It is a method of testing fragments called cell-free DNA (cfDNA) in a pregnant woman's blood. These fragments are derived from both mother's and placenta's cells. DNA of the placenta cells is indistinguishable from the fetus's, thus analyzing this cfDNA offers an opportunity for early detection of genetic abnormalities harmlessly., ${ }^{9,10}$ There is a protein gene in the placenta cells, namely Hydroxysteroid 11-Beta Dehydrogenase 2 (HSD11B2) gene, which encodes a placental enzyme. None other, 11ß-hydroxysteroid dehydrogenase type-2 (11 $\beta$-HSD2), a fetal barrier against glucocorticoids. Glucocorticoids themselves are stress hormones. One of the known causes of IUGR is the lack of this enzyme. ${ }^{11,12}$

Therefore, this literature review discusses whether changes in the HSD11B2 gene significantly affect the $11 \beta$-HSD2 enzyme and cause IUGR. Screening for HSD11B2 gene changes through NIPT screening could be a possible early detection parameter to prevent the progression of IUGR.

\section{Data collection}

In this review, literature was searched through Pubmed, Proquest, and EBSCO database using the keyword "Intrauterine Growth Restriction" or "Fetal Growth
Retardation" or "Growth Retardation, Intrauterine" or "Intrauterine Growth Retardation" or "Fetal Growth Restriction" or "IUGR" and "HSD11B2". Inclusion criteria used in this review were studies that conduct an alteration of HSD11B2 gene in IUGR patients and original articles published in the last 10 years. Exclusion criteria used were studies published not in English; and abstract, reviews, case reports, or book sections. Literature obtained from database search was firstly screened based on title and abstract. We got 15 potential pieces of literature from the first selection. Potential literature was further assessed by reading its full text. In the end, we concluded 4 articles to be included in this review.

\section{IUGR}

IUGR is a condition that affects fetal growth rate below the normal potential according to certain races and gender of the fetus. The birth weight of a baby does not determine the condition for IUGR. However, both IUGR and small for gestational age (SGA) are interchangeably used, though these terms are slightly different. Infants with IUGR possibly have the ideal weight according to certain gestation age, but at an early age may have suffered from perinatal disturbance resulting in growth deceleration. The term IUGR is often used in infants with clinical signs of malnutrition. Meanwhile, SGA is used to diagnose an infant's condition whose birth weight is less than the 10th percentile according to certain gestational age or normal degree of the population's growth chart without considering any possible delayed growth in the uterus. Early gestational age increases the risk for the infant to suffer from IUGR. ${ }^{2}$

IUGR can be classified into two different types, asymmetrical (type 1) and symmetrical (type 2). The asymmetrical type occurs in the third semester and is caused by placental insufficiency regarding its nutrition and oxygen supply function critical for fetus growth. Nearly $70-80 \%$ of IUGR cases are asymmetrical. Whereas the symmetrical type, which is high in risk for mortality and morbidity, is associated with the fetus's genetic defects or intrinsic infection. The etiology of IUGR can vary, such as maternal, fetal, genetic, or placental factors. Diverse maternal factors, such as socioeconomic factors, maternal health, infection, and behavioral, can cause IUGR. Yet fetal factors such as congenital infections, aneuploidies, and genetic syndromes also play a role. Abnormality of the placenta, including genetic alteration, has become an important cause of IUGR. ${ }^{2,13}$ 


\section{HSD11B2 as a placental enzyme coding gene}

The placenta plays a major role in maintaining fetal growth as the main organ to keep blood flow as well as nutrients, oxygen, and other solutes in it from the maternal circulation. ${ }^{2}$ It is known that IUGR infants have some placental abnormalities such as infection, circulation disturbance, and genetic defect. Placental tissue expresses some gene called the HSD11B2 that prominently encodes the production of the $11 \beta$-HSD2 enzyme. This enzyme acts as the barrier for the fetus from cortisol to enter the placenta through the mechanism of converting glucocorticoid cortisol to its inactive form, the cortisone. ${ }^{14}$ Exposure to this stress hormone could cause a reduction in cell proliferation and lead to growth restriction. ${ }^{15}$ In the placenta, $11 \beta$-HSD2 enzyme protects the placental cells from pro-apoptotic and growth inhibition effects of cortisol during the embryo growth. Any mutation of the HSD11B2 could result in mineralocorticoid excess and hypertension. Alteration of the gene may be associated with the process called DNA methylation, by which there will be a methyl group added to the $5^{\text {th }}$ carbon chain of the existing DNA molecule supported by the DNA methyltransferase enzyme. ${ }^{14}$ Methylation is an epigenetic regulation that occurs due to the stress of some environmental conditions. It plays a principal role in managing gene activities. Epigenetic modification of the HSD11B2 gene may lead to a deficiency of 11ß-HSD2 enzyme, the placental enzyme. Given that some studies show IUGR infants are proven to have HSD11B2 gene methylation, downregulation of $11 \beta-\mathrm{HSD} 2$ enzyme is related to growth restriction. ${ }^{11}$

The 11 $\beta$-HSD2 enzyme has a crucial role in protecting fetus from the potency of glucocorticoid toxicity obtained from maternal endogenous steroid production or any steroid drug consumption. Hence, some conditions in a particular resulting reduction of the 11ß-HSD2 enzyme availability might raise the level of glucocorticoid entering the fetus blood via placenta circulation in consequence of lower glucocorticoid metabolism that used to transform the active to inactive form reducing its toxicity. One of the negative impacts of excess glucocorticoid in fetus blood is a disruption in the growth process and the worst may risk the fetus to suffer from IUGR. ${ }^{16}$

\section{The need for early detection test in IUGR}

IUGR in the fetus needs immediate detection to reduce its worse progression. A diagnostic method used is the symphyseal fundal height (SFH), which is plotted toward some population chart. If the result shows it is likely to be an IUGR, the follow-up examination using Doppler ultrasound needs to be done at the third trimester. The ultrasound examination will be used to assess the abdominal circumference and estimated fetal weight. Sequences of the diagnostic methods were reported to be inaccurate and can still be affected by many external factors. ${ }^{5}$ Assessment results may not be consistent and have poor clinical predictive value in various women. Furthermore, the diagnostic methods can be done since the second trimester (between 13-28 weeks of pregnancy), which is too late to do early detection. ${ }^{6,17}$ Doppler ultrasound is not advised to be done in the first trimester due to its possible thermal effects on the fetus. ${ }^{18-20}$ A study shows that the screening methods to assess IUGR infants have sensitivity as much as $61 \%$ and specificity of $56 \%$. The study suggests that diagnostic value could be enhanced by combining the fetus's routine measurement and using a placental biomarker. ${ }^{5}$

Some studies exhibit the importance of $11 \beta-H S D 2$ related to the incidence of IUGR. Thus, the correlation between the two could be used to develop a new biomarker for the early detection of IUGR cases. ${ }^{5}$

\section{IUGR vs. HSD11B2 gene alteration}

Alteration in DNA has a role in every disease. ${ }^{21}$ HSD 11B2 alteration may vary and some may affect the fetal growth by a series of mechanisms. Defects in the HSD11B2 gene lead to the deficiency of the 11ß-HSD2 enzyme which plays a major role in converting cortisol to inactive cortisone and protects the mineralocorticoid receptor from being activated by the circulating cortisol. Thus, the absence of the 11ß-HSD2 enzyme may result in apparent mineralocorticoid excess (AME). ${ }^{22}$ A previous study explained structural alteration of $H S D 11 B 2$, allows the formation of inactive dimer and alter coenzyme binding, generating severe AME. Severe AME is known to be correlated with IUGR since the deficiency of the 11 $\beta$-HSD2 enzyme allows excessive amounts of maternal glucocorticoid to cross the placental barrier resulting in inhibition of fetal growth. ${ }^{23}$

\section{IUGR vs. HSD11B2 gene methylation}

A study evaluated the relationship between HSD11B2 methylation in the $\mathrm{CpG}$ region of HSD11B2 gene promoter with infant and mother characteristics. This study used 185 healthy newborn placentas, and they were analyzed with 
bisulfite pyrosequencing and qRT-PCR quantitatively. The result showed that $H S D 11 B 2$ methylation significantly increased in IUGR infants than in controls. $(p=0.007) .{ }^{11}$

Another study evaluated the HSD11B2 gene in 22 IUGR patients and 22 healthy patients as control. This study showed that HSD11B2 methylation in $4 \mathrm{CpG}$ regions significantly increased in IUGR patients than controls with mean difference values $2.31,2.36,1.64$, and $2.18 \%$, respectively. $(\mathrm{p}=0.020,0.021,0.027$, and 0.029$){ }^{24}$

Meanwhile, other study evaluated the methylation of HSD11B2 gene in 80 IUGR placentas and 101 healthy placentas as controls using the PCR method. This study showed that methylation HSD11B2 gene in $4 \mathrm{CpG}$ regions significantly increased in IUGR patients than controls $(\mathrm{p}=0.003) .{ }^{25}$ Figure 1 shows the correlation between mean methylation rate and clinical status of IUGR.

\section{IUGR vs. HSD11B2 gene expression}

Significant underexpression of the HSD11B2 gene in IUGR groups compared to the control groups. (ln 2a: 1.28; $p<0.05)$. Before $33^{\text {rd }}$ week gestation, there was no significant difference between the IUGR group and the control group but after $33^{\text {rd }}$ week gestation, HSD11B2 gene activity significantly decreased compared to the control group. The statistical value for decreased $H S D 11 B 2$ gene activity was similar to the IUGR subgroup with a gestational age of 3337 weeks and a gestation age of more than 37 weeks $(\ln 2 \mathrm{a}$ : $1.19 ; p<0.05 ; \ln 2 \mathrm{a}: 1.31 ; p<0.05) .{ }^{14}$

A study showed that mRNA HSD11B2 was significantly under-expressed in IUGR groups $(p=0.003)$. This study used 80 IUGR placenta, 101 healthy placentas as controls, and they were analyzed by PCR. ${ }^{24}$ The summary of each study characteristics are shown in Table 1.

\section{NIPT as a potential IUGR screening test}

Alteration in the $H S D 11 B 2$ gene may be associated with the interruption of $11 \beta-H S D 2$ enzyme production. Thus, the correlation between the two could be used to develop a new biomarker for the early detection of IUGR cases. The sample of HSD11B2 gene can be obtained from NIPT. NIPT is a screening method to examine chromosomal and gene defects in a fetus as early as 10 weeks gestation by a simple procedure of drawing maternal blood. During pregnancy, some fetal cells are found in maternal circulation, including fetal trophoblasts, lymphocytes, granulocytes, nucleated erythrocytes, and platelets. ${ }^{26}$ The highest expression of the HSD11B2 gene is known to exist in the syncytiotrophoblast, thus the isolation of this gene from the maternal circulation is reasonable to perform. ${ }^{27}$ Moreover, there is a higher amount of cell-free fetal DNA (cffDNA), fragments of DNA released from the apoptotic placental cells, than the fraction of the fetal cells in maternal circulation. These fragments of cffDNA are later extracted from the maternal blood and will be used to evaluate fetal genetic material, making it more reliable to conduct NIPT as a screening method to carry out early detection of IUGR. ${ }^{26,28} \mathrm{~A}$ previous study mentioned the implications of cff-DNA detection for a scope of pregnancyrelated complications, for instance, IUGR, preeclampsia, hyperemesis gravidarum and placenta previa. ${ }^{29}$ Compared to the other diagnostic methods, particularly the procedure of collecting the sample such as chorionic villus sampling (CVS) which is done transabdominally or transcervically, NIPT is considered to be non-invasive since it doesn't make any direct contact to the fetal environment, thus lower the risk of causing potential harm to the fetal growth and chance of miscarriage. ${ }^{26}$ It has obtained increasing clinical acceptance and believed as a genetic diagnostic with extensive applications. ${ }^{30}$

This study still has several limitations, including a lack of literature that describes the association of $H S D 11 B 2$ gene with the incidence of IUGR patients, and several studies only reported significant testing without diagnostic accuracy (AUC values). Therefore, when conducting diagnostic analysis, we recommend researchers apply ROC curve analysis and AUC statistics rather than a single diagnostic measure such as sensitivity and specificity that are subjectively reliant on the level of cutoffs determined. We also recommend researchers explore more about the association of $H S D 11 B 2$ gene and the incidence of IUGR.

\section{Conclusion}

We observed several studies that reported an increase in DNA methylation and under-expression of the HSD11B2 gene in IUGR infants upon our literature searches. This study showed that HSD11B2 gene changes could be a promising diagnostic parameter in preventing the progression of infants with IUGR. NIPT would be the prospective method to detect those alterations, specifically in the HSD $11 B 2$ gene. 


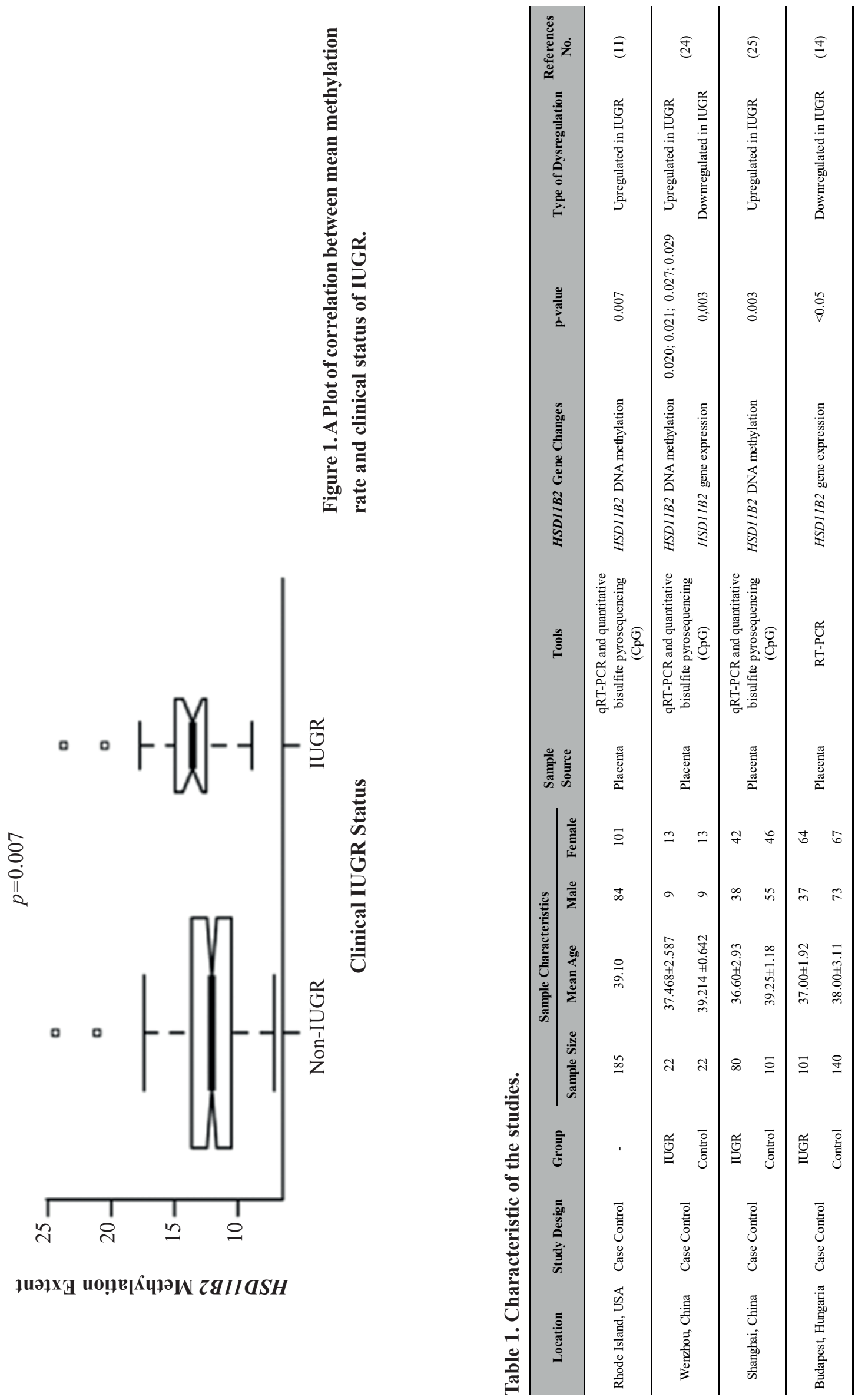




\section{References}

1. Giuliano N, Annunziata ML, Tagliaferri S, Esposito FG, Imperato OC, Campanile M, et al. IUGR management: new perspectives. J Pregnancy. 2014; 2014: 620976. doi: 10.1155/2014/620976.

2. Sharma D, Shastri S, Sharma P. Intrauterine growth restriction: antenatal and postnatal aspects. Clin Med Insights Pediatr. 2016; 10: 67-83.

3. Unterscheider J, O'Donoghue K, Daly S, Geary MP, Kennelly MM, McAuliffe FM, et al. Fetal growth restriction and the risk of perinatal mortality-case studies from the multicentre PORTO study. BMC Pregnancy Childbirth. 2014; 14: 63. doi: 10.1186/1471-239314-63.

4. Hasmasanu M, Bolboaca S, Baizat M, Drugan T, Zaharie G. Neonatal short-term outcomes in infants with intrauterine growth restriction. Saudi Med J. 2015; 36(8): 947-53.

5. Poljak B, Agarwal U, Jackson R, Alfirevic Z, Sharp A. Diagnostic accuracy of individual antenatal tools for prediction of small-forgestational age at birth. Ultrasound Obstet Gynecol. 2017; 49(4): 493-9.

6. Lee S, Walker SP. The role of ultrasound in the diagnosis and management of the growth restricted fetus. Australas J Ultrasound Med. 2010; 13(3): 31-6.

7. Ernst SA, Brand T, Reeske A, Spallek J, Petersen K, Zeeb H. Carerelated and maternal risk factors associated with the antenatal nondetection of intrauterine growth eestriction: a case-control study from Bremen, Germany. Biomed Res Int. 2017; 2017: 1746146. doi: 10.1155/2017/1746146.

8. Pinto A, Pinto F, Faggian A, Rubini G, Caranci F, Macarini L, et al. Sources of error in emergency ultrasonography. Crit Ultrasound J. 2013; 5(Suppl 1): S1. doi: 10.1186/2036-7902-5-S1-S1.

9. Dondorp W, de Wert G, Bombard Y, Bianchi DW, Bergmann C, Borry $\mathrm{P}$, et al. Non-invasive prenatal testing for aneuploidy and beyond: challenges of responsible innovation in prenatal screening. Eur $\mathrm{J}$ Hum Genet. 2015; 23: 1438-50.

10. Skrzypek H, Hui L. Noninvasive prenatal testing for fetal aneuploidy and single gene disorders. Best Pract Res Clin Obstet Gynaecol. 2017; 42: 26-38.

11. Marsit CJ, Maccani MA, Padbury JF, Lester BM. Placental 11-beta hydroxysteroid dehydrogenase methylation is associated with newborn growth and a measure of neurobehavioral outcome. PLoS One. 2012; 7(3): e33794. doi: 10.1371/journal.pone.0033794.

12. Wynne-Edwards KE, Edwards HE, Hancock TM. The human fetus preferentially secretes corticosterone, rather than cortisol, in response to intra-partum stressors. PLoS One. 2013; 8(6): e63684. doi: 10.1371/journal.pone.0063684.

13. Albu AR, Anca AF, Horhoianu VV, Horhoianu IA. Predictive factors for intrauterine growth restriction. J Med Life. 2014; 7(2): 165-71.

14. Börzsönyi B, Demendi C, Pajor A, Rigó J Jr, Marosi K, Agota A, et al. Gene expression patterns of the 11ß-hydroxysteroid dehydrogenase 2 enzyme in human placenta from intrauterine growth restriction: the role of impaired feto-maternal glucocorticoid metabolism. Eur J Obstet Gynecol Reprod Biol. 2012; 161(1): 12-7.

15. Burton GJ, Fowden AL. The placenta: a multifaceted, transient organ. Philos Trans R Soc Lond B Biol Sci. 2015; 370(1663): 20140066. doi: 10.1098/rstb.2014.0066.

16. Stirrat L, Just G, Homer N, Andrew R, Norman J, Reynolds R. Glucocorticoids are lower at delivery in maternal, but not cord blood of obese pregnancies. Sci Rep. 2017; 7(1): 10263. doi: 10.1038/s41598-017-10266-5.

17. Grow by WebMC [Internet]. Fetal Doppler [cited 11 September 2021]. Available from: https://www.webmd.com/baby/fetal-doppler\#1.

18. Bhoil R, Kaushal S, Sharma R, Kaur J, Sharma T, Thakur R, et al. Color Doppler ultrasound of spiral artery blood flow in mid first trimester (4-8 weeks) in cases of threatened abortion and in normal pregnancies. J Ultrason. 2019; 19(79): 255-60.

19. Sheiner E, Abramowicz JS. A symposium on obstetrical ultrasound: is all this safe for the fetus? Clin Obstet Gynecol 2012; 55(1): 188-98.

20. Helmy S, Bader Y, Koch M, Tiringer D, Kollmann C. Measurement of thermal effects of Doppler ultrasound: an in vitro study. PLoS One. 2015; 10: e0135717. doi: 10.1371/journal.pone.0135717.

21. Sari B, Zahra A, Tasti G, Maritska Z. Healing the fundamental unit of heredity (gene therapy): current perspective and what the future holds. Mol Cel Biomed Sci. 2021; 5(2): 62-7.

22. Yau M, Haider S, Khattab A, Ling C, Mathew M, Zaidi S, et al. Clinical, genetic, and structural basis of apparent mineralocorticoid excess due to $11 \beta$-hydroxysteroid dehydrogenase type 2 deficiency. Proc Natl Acad Sci USA. 2017; 114(52): E11248-56.

23. Bertulli C, Hureaux M, De Mutiis C, Pasini A, Bockenhauer D, Vargas-Poussou R, et al. A rare cause of chronic hypokalemia with metabolic alkalosis: case report and differential diagnosis. Children (Basel). 2020; 7(11): 212. doi: 10.3390/children7110212

24. Zhao Y, Gong X, Chen L, Li L, Liang Y, Chen S, et al. Site-specific methylation of placental HSD11B2 gene promoter is related to intrauterine growth restriction. Eur J Hum Genet. 2014; 22(6): 73440.

25. Xiao X, Zhao Y, Jin R, Chen J, Wang X, Baccarelli A, et al. Fetal growth restriction and methylation of growth-related genes in the placenta. Epigenomics. 2016; 8(1): 33-42.

26. Norwitz ER, Levy B. Noninvasive prenatal testing: the future is now. Rev Obstet Gynecol. 2013; 6(2): 48-62.

27. Kosicka K, Siemiątkowska A, Główka FK. 11ß-hydroxysteroid dehydrogenase 2 in preeclampsia. Int J Endocrinol. 2016; 2016: 5279462. doi: 10.1155/2016/5279462.

28. Woolcock J, Grivell R. Noninvasive prenatal testing. Aust Fam Physician. 2014; 43(7): 432-4.

29. Sifakis S, Koukou Z, Spandidos DA. Cell-free fetal DNA and pregnancy-related complications (review). Mol Med Rep. 2015; 11(4): 2367-72.

30. Yin A, Peng C, Zhao X, Caughey B, Yang J, Liu J, et al. Noninvasive detection of fetal subchromosomal abnormalities by semiconductor sequencing of maternal plasma DNA. Proc Natl Acad Sci USA. 2015; 112(47): 14670-5. 